\title{
Identification and Evaluation of Coagulation Promotors on Surface of Medical Materials
}

\author{
Akari Goto, Kunihiko Ishihara
}

\begin{abstract}
During hemodialysis treatment, many problems such as residual blood, thrombocytopenia, coagulation in the blood circuit occur due to the patient's blood contacting with various materials. Then in this paper, we will propose to evaluate the thrombin activity caused by contact between blood and material as an evaluation method of the antithrombotic medical material.

In the previous study, as the objective of elucidation of the blood coagulation mechanism, thrombin-like activity remained on the surface of the medical materials was measured and compared in order to evaluate the difference of materials for the blood coagulation activation which is caused in the case of the blood contacting the medical materials outside the living body.

In the previous study, thrombin-like activity is confirmed for five kinds of materials such as polystyrene, polymethylpentene, polyoropylene, tetrafluoroethylene, perfluoroalkoxyethylene and glass. In the present study, it was clarified that the coagulation promoter adsorbed on the surface of the material was thrombin due to the experiment using anti-thrombin antibody.
\end{abstract}

Index Terms - Blood coagulation reaction. Hemodialysis therapy, Thrombin, Materials

\section{INTRODUCTION}

Hemodialysis is a treatment, a procedure that is performed by utilizing an artificial kidney where the blood is led through a blood circuit.

A coagulation reaction is initiated when blood flows out of blood vessels. The same reaction occurs in the blood circuit during hemodialysis.

During treatment, a synthetic anti-thrombin drug such as argatroban or an anticoagulant, like heparin or low-molecular weight heparin must be used to suppress these coagulation reactions. The treatment was carried out smoothly.

However, problems due to blood coagulation reactions often arise due to the use of anticoagulants during dialysis and blood coagulation complications in hemodialysis patients.

These problems include residual blood in the circuit during dialysis, and thrombocytopenia caused by dialysis. This residual blood and coagulation in the blood circuit are caused by activities of the white blood cells and, platelets, types of biomaterials that come into contact, the contact area, and the charged state respectively. Attention has been paid to the interaction between platelets and adhesive proteins, such as fibrinogen and vitronectin. We evaluated whether the coagulation promoter was adsorbed on the surface of medical materials coated on polymers. An activity similar to that of thrombin has been confirmed so far although we could not identify the same $e^{2,3}$ ).

In previous studies, we confirmed thrombin activity by color development quantification using the color development synthetic substrate S-2238 to identify the substances adsorbed on the material surface.
Therefore, the substances confirmed so far were named based on their thrombin-like activity, and we conducted the experiment accordingly.

Thrombin activity was confirmed on the material surface after washing when the heparinized blood remained in a test tube made of various polymer materials used in all the medical devices. There was a strong correlation between solidification time and thrombin-like activity; that is, shorter the solidification time, higher was the residual thrombin-like activity $^{3}$ ).

As a result, almost no residual thrombin-like activity was observed in the same experiment when decalcification anticoagulants such as EDTA and sodium citrate were included.

In this study, we confirmed the role of a coagulation promoter that was considered to be a thrombin-like substance, as mentioned in a previous study. The activation of blood coagulation factors due to contact between blood and foreign matter and their effects were evaluated accordingly.

\section{RESEARCH METHOD}

\section{A. Object to be considered}

The present study was performed after providing sufficient information about the purpose of the research, protection of personal information, and voluntary cooperation from the participants with approval from the Ethics Review Board of Tokushima Bunri University.

\section{B. Method}

In this study, the quantitative evaluation of residual thrombin on the surface of each polymer material was performed to reveal that the thrombin-like activity, which was confirmed on polymer materials used in the medical field, was in fact that of thrombin.

Further, anti-thrombin rabbit antiserum was combined with residual thrombin, and color development was quantified using the biotin-avidin method.

Specific biotinylated anti-thrombin antibody rabbit serum was reacted with thrombin, which was the target antigen in the sample, and the primary antibody and enzyme reagents were reacted using a secondary antibody as a link. As a result, the antigen primary antibody -secondary antibody- peroxidase complex was formed through the high-affinity binding force of biotin and streptavidin.

After the addition of the peroxidase substrate, the color of peroxidase developed in the sample. The color intensity was assessed to measure the amount of thrombin present in the sample, which was proportional to the amount of thrombin present in the sample.

Glass $(\varphi 10 \times 75 \mathrm{~mm})$, polyvinyl chloride:PVC $(\varphi 8 \times 100 \mathrm{~mm})$, polymethylpentene : PMP $(\varphi 16.4 \times 100 \mathrm{~mm})$, polypropylene: PP $(\varphi 16.5 \times 100 \mathrm{~mm})$, polystyrene: PS 
$(\varphi 14.5 \times 83 \mathrm{~mm})$, and per fluoro alkoxy: PFA $(\varphi 10 \times 140 \mathrm{~mm})$ were used as test tube materials, and the experiment was performed five times for each test tube.

The pump segment part of the blood circuit set (NIKKISO Blood Circuit NV-Y/Z) for dialysis made of PVC was used as the PVC test tube.

The experimental procedure was as follows.

1. Blood was collected from the patients after obtaining informed consent; and immediately after the collection procedure, an anticoagulant was added to the blood sample. Heparin (Mochida Pharmaceutical Co. Ltd.) was used as an anticoagulant, and 10 units of heparin were required for $1 \mathrm{~mL}$ of blood.

The experiment was performed immediately according to the procedure described in (Step 2) using heparinized blood.

Blood samples that were not used immediately and were kept over time were not used. Complete anonymized blood was used in this experiment.

2. The heparinized blood $(1 \mathrm{~mL})$ in a test tube made of polymer material was kept inside a constant-temperature bath heated to $37{ }^{\circ} \mathrm{C}$ for $60 \mathrm{~min}$. The test tube was washed with saline.

Biotinylated anti-thrombin antibody rabbit serum $(1 \mathrm{~mL})$ diluted 50 times with saline was placed in a test tube after cleaning. It was kept inside a constant-temperature bath heated to $37^{\circ} \mathrm{C}$ for $10 \mathrm{~min}$, and the test tube was washed with saline.

The set temperature of $37^{\circ} \mathrm{C}$ in the constant temperature bath described in (Step 2) simulated the body temperature.

3. Peroxidase labelled streptavidin (Streptavidin RUO, Dako) diluted 40 times with saline $(1 \mathrm{ml})$ was placed in a test tube. It was maintained inside a constant temperature bath and heated to $37{ }^{\circ} \mathrm{C}$ for $30 \mathrm{~min}$. The test tube was washed with saline.

The set temperature of $37{ }^{\circ} \mathrm{C}$ for the constant temperature bath described in step (2) simulated the body temperature.

4. Substrate solution (bis(3-ethylbenzothiazoline-6-sulfonic Acid) diammonium salt. ) (1 mL) was placed in the test tube after cleaning, and was allowed to stand for $20 \mathrm{~min}$ at room temperature .

The substrate reacted and showed a blue color.

5. After standing still, the reaction solution in each test tube was placed in a cuvette to measure its absorbance.

Absorbance was measured using a spectrophotometer (Hitachi 201) at a wavelength of $405 \mathrm{~nm}$.

6. The test tube, cuvette, and blood sample after the experiment were stored in a medical waste container, outsourced to a specialist, and discarded accordingly.

\section{Statistical analysis}

Statistical differences in the amount of residual thrombin between the various materials were confirmed using t-tests. $\mathrm{P}<0.05$, was considered statistically significant.

\section{RESULTS}

Thrombin activity was observed in each test tube when blood containing anticoagulant heparin was adsorbed on test tubes of different types of materials.

In each material, the amount of thrombin was observed. For example, for glass, it was $0.95 \mathrm{pkat} / \mathrm{mL}$; , for PS, $0.7 \mathrm{pkat} /$
$\mathrm{mL}$; for PP, $1.95 \mathrm{pkat} / \mathrm{mL}$; for, PVC, $2.65 \mathrm{pkat} / \mathrm{mL}$; for PMP, 2.95 pkat $/ \mathrm{mL}$; for PFA, it was $3.10 \mathrm{pkat} / \mathrm{mL}$.

From the surface of the polymer material that had excellent antithrombotic properties and was used as a biomaterial accordingly, it was confirmed that the thrombin amount of the polymer material was larger than that of glass, even after washing it with saline.

In the present experiment, it was clarified that PS had a low amount of residual thrombin; consequently, thrombin did not easily adhere to the surface of the material. It was also confirmed that a significant difference existed between the glass and the four types of polymer materials (except PS).

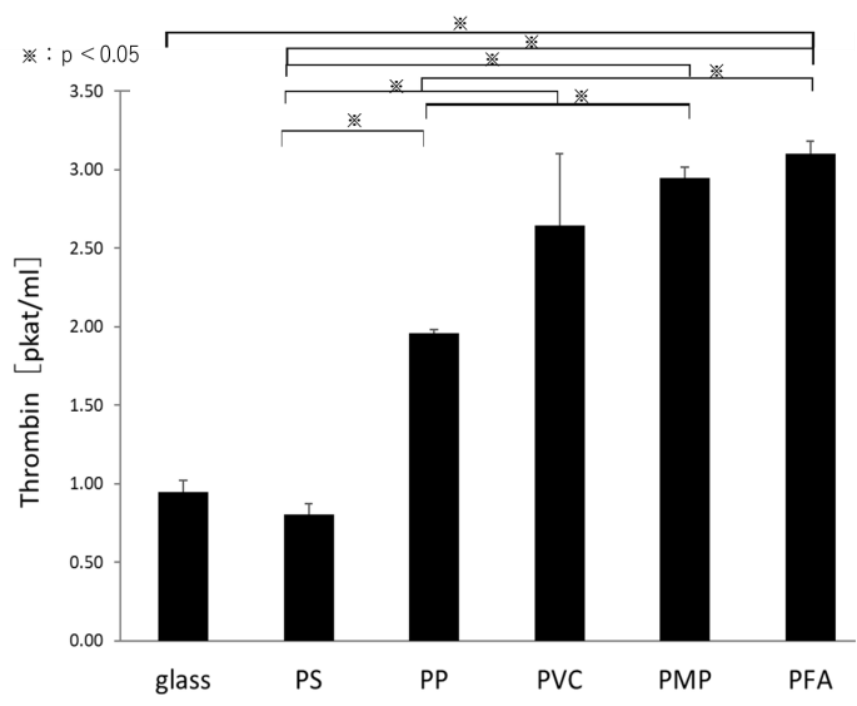

Fig.1 Comparison of residual thrombin on surface of different materials

\section{CONSIDERATION}

The thrombin-like activity adsorbed on the material surface, which was confirmed in previous research, was thrombin itself, which was confirmed in our study.

Furthermore, the thrombin-like activity revealed in previous studies was compared with the residual thrombin amount determined in this study (Fig. 2).

It had been considered that no significant difference existed between the residual thrombin amount and thrombin activity in any material. However, a significant difference between the two was observed for glass, PP, and PMP. In glass, which contributed to the enhancement of the endogenous coagulation reaction, it was confirmed that the amount of residual thrombin was lower than that of the thrombin-like activity.

It is possible that the adsorbed substance may have been released on the glass surface during the experiment. However, substances similar to thrombin, other than thrombin, and other coagulation promoters, are easily adsorbed. Therefore, it is considered that the thrombin analogue adsorbs to the material and contributes to the enhanced coagulation reaction.

The amount of residual thrombin in PP and PMP was superior to that of thrombin-like activity. Thrombin was more 
easily adsorbed than thrombin-like substances, depending on the type of polymer material.

Under treatment that involves extracorporeal circulation, including hemodialysis, blood is activated, and thrombin is produced due to contact between heparinized blood and polymer materials. The thrombin produced may increase blood coagulation without binding to anti-thrombin (AT).

In this case, the produced thrombin adsorbs to the material surface of the blood circuit used in treatment procedures such as hemodialysis. However, it can be considered that the activity parts are not materials but states of the materials that are exposed to blood (Fig. 3) if the thrombin adsorbs on the material surface through the heparin binding site (Fig. 3).

Therefore, even if heparin is used as an anticoagulant, AT cannot bind to the thrombin adsorbed on the material. This is because a heparin-binding site is adsorbed on the material surface.

Additionally, the anticoagulant effect may not be caused despite administering anticoagulants such as heparin.

At the same time, it is considered that the coagulation reaction is enhanced because the thrombin active group is exposed on the blood side.

Many modules are involved in the function of thrombin, which changes its structure due to the action of amino acid side chains and functional regulators ${ }^{4}$ ).

The structure and activity of thrombin are considered to change upon adsorption onto the material surface. However, the effect of residual thrombin and thrombin active groups on blood coagulation time has not yet been verified.

Further verification is needed in the future; for the treatment that involves extracorporeal circulation of blood because increased blood coagulation response in pathways in the blood circuit is caused by thrombin adsorption on the material surface.

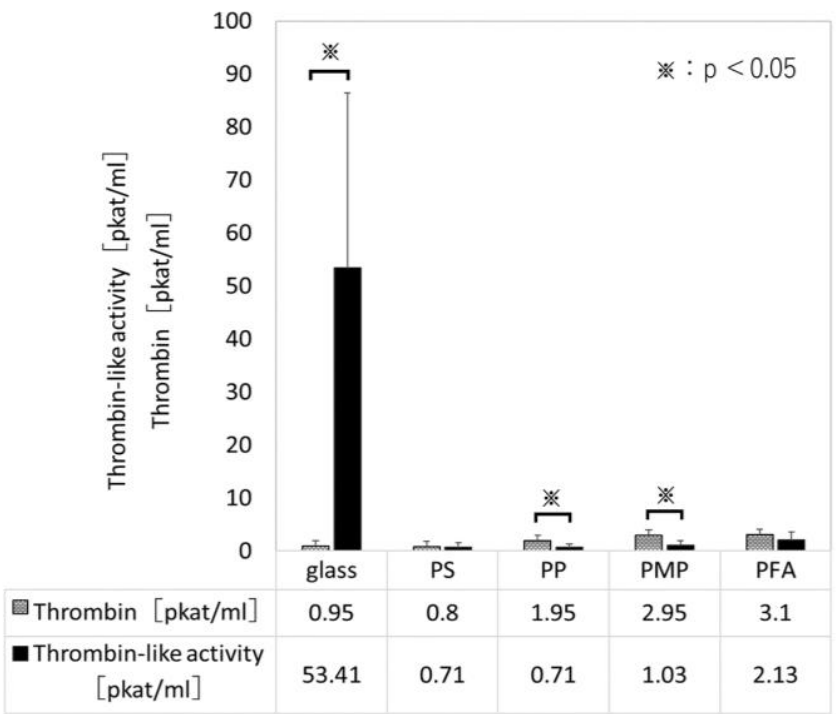

Fig.2 Comparison of residual thrombin with thrombin-like activity

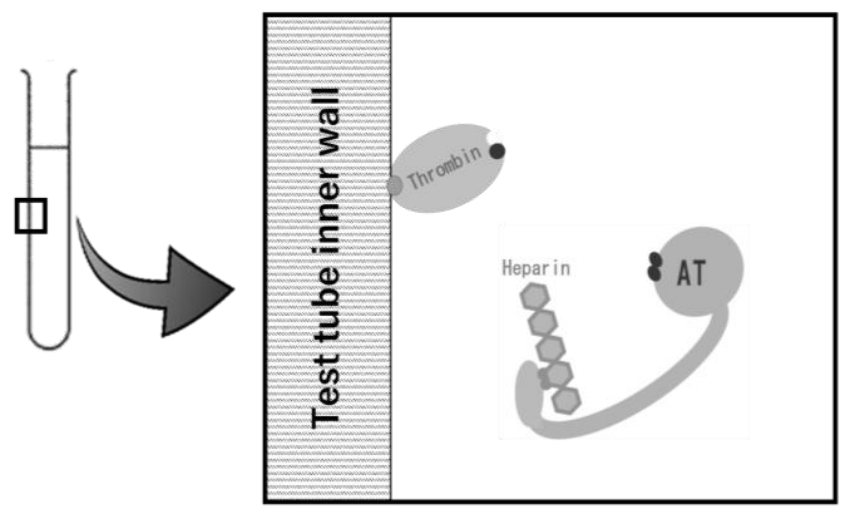

Fig.3 Blood coagulation activity due to thrombin adsorbed on material surface

\section{CONCLUSION}

The present study confirmed that the thrombin -like activity of the medical materials observed in the previous study was thrombin.

It also revealed that blood coagulation by thrombin adsorption on the inner surface of the circuit can be caused, in treatment with extracorporeal circulation during hemodialysis, even if we use anticoagulants such as heparin. This poses a significant problem.

Although anticoagulants such as heparin are used, the treatment may be administered without suppressing or avoiding the blood coagulation reaction.

It is necessary to suppress the blood coagulation reaction completely by using anticoagulant-chelating calcium, such as EDTA and sodium citrate, or by using substances with anticoagulant effects, such as AnnexinV, that prevent blood coagulation by covering the surface of the phospholipid membrane.

However, the use of decalcifying agents may interfere with treatments such as hemodialysis therapy ${ }^{5}$ ). Thus, the development of appropriate decalcifying agents suitable for the human body is desirable.

\section{REFERENCES}

[1] S.Asakura, "Dialytic therapy and blood clotting fibrinolysis. Hematologic problems in the clinical dialysis", June extra number issue 1998, Vol.14, No. 8, dialytic therapy Japan, 1998, pp.25-38.

[2] A.Goto, "Study on effect of materials giving it to blood clotting reaction", Thesis for doctorate of Hiroshima international university, 2011.

[3] A. Gotow and S. Fukunaga, "Effects of materials on the Blood Coagulation", Structure and Fuction, Vol.10, No.2, 2012, pp.80-85.

[4] K. Suzuki, "Thrombin functional module", Thrombus hemostatic magazine, Vol.27, No.5, 2016, pp.563-574.

[5] H. Morimatsu, "Citric acid as the anticoagulant in the kidney alternative therapy", Japanese intensive care medical society magazine, Vol. 20, No. 4, 2013, pp.577-579.

[6] A. Okuda, S. Uchino, T. Shibasaki, R. Iwatani, A. Hirazuka, K. Saito and M. Takinami, "Enforcement experience of the sustained haemodialysis with citric acid, Japanese intensive care medical society magazine, Vol. 20, No. 4, 2013, pp.653-654. 
Akari Goto was born in 1984 in Tonosho, Kagawa Prefecture Japan. She received a bachelor's degree (clinical engineering) from Hiroshima International University in 2007. She got a master's degree in 2009 and earned the Ph.D. degree in Medical Engineering from The Hiroshima International University in 2012.

She became a lecturer at Tokushima Bunri University in 2009. And she has been a teacher since 2011 .

Kunihiko Ishihara was born in 1947 in Kurashiki City, Okayama Prefecture Japan. He received the B.S. degree from Kobe University in 1969. He got a master's degree in Kobe University in 1971 and earned the Ph.D. degree in Engineering from The Osaka University in 1986.

He worked in Kawasaki Heavy Industry Co. Ltd. as an Mechanical Engineer for 33 years. After that he became a Professor of The University of Tokushima in 2004. He had been studying the vibration and noise control, above all he studied the flow induced vibration and noise problems. He has authored or co-authored over 100 technical journal and over 50 conference papers. He is a fellow of JSME (Japan Society of Mechanical Engineers) now. He is a Professor of Tokushima Bunri University. He teaches a mechanical field subjects for students. 